\title{
Application of Cyclostationarity to Joint Signal Detection, Classification, and Blind Parameter Estimation
}

\author{
Invited Paper \\ ${ }^{1}$ Octavia A. Dobre, ${ }^{2}$ Robert Inkol, and ${ }^{2}$ Sreeraman Rajan \\ ${ }^{1}$ Memorial University of Newfoundland, St. John's, NL A1B 3X5, Canada \\ ${ }^{2}$ Defence Research and Development Canada, 3701 Carling Avenue, Ottawa, Ontario K1A 0Z4, Canada \\ Emails: ${ }^{1}$ odobre@mun.ca, ${ }^{2}$ robert.inkol@drdc-rddc.gc.ca, ${ }^{2}$ sreeraman.rajan@drdc-rddc.gc.ca
}

\begin{abstract}
This paper provides a brief overview of selected achievements by our group concerning cyclostationarity-based joint signal detection, classification, and blind parameter estimation [1]-[9]. These developments have important applications in signal intelligence, spectrum surveillance and management, and are highly relevant to spectrum sensing and awareness in cognitive radio.
\end{abstract}

\section{INTRODUCTION}

The cyclostationarity properties of signals have been widely investigated for communications, biomedical, radar, and sonar applications. These properties can be exploited for various purposes, including signal detection, classification, parameter estimation, synchronization, and equalization [1]-[15]. Communication signals exhibit cyclostationarity associated with the symbol period, carrier frequency, pilot position, etc. [1]-[15]. This paper presents results on the first-, second-, and higher-order cyclostationarity-based signal features associated with amplitude modulation (AM), frequency shift keying (FSK), single carrier linearly digitally modulated (SCLD), such as phase shift keying (PSK) and quadrature amplitude modulation (QAM), cyclically prefixed SCLD (CP-SCLD), and generic and standard orthogonal frequency division multiplexing (OFDM) signals. We also show how these features can be used for joint signal detection, classification, and blind parameter estimation.

The rest of the paper is organized as follows. Section II introduces models for the major signal classes. Section III defines first-, second-, and higher-order signal cyclostationarity and identifies the distinctive cyclostationarity-based signal features that correspond to each signal class. In Section IV we show how these features can be exploited for joint signal detection, classification, and blind parameter estimation. Numerical and simulation results are presented in Section V, and conclusions are drawn in Section VI. In addition, the definitions of time domain parameters of signal cyclostationarity and their sample estimates are given in Appendix A. Finally, a cyclostationarity test used for decision making in signal detection and classification is described in Appendix B.

\section{Signal Models}

Using either the estimates or a priori knowledge of bandwidth and carrier frequency, the received signal can be extracted and its signal-to-noise ratio maximized by down-conversion and baseband filtering. The resulting signal can be expressed as

$r(t)=s(t)+w(t)$,

where $s(t)$ can be an AM, FSK, SCLD, CP-SCLD, or OFDM signal, and $w(t)$ is the additive zero-mean Gaussian noise.

For an AM signal, $s(t)$ can be expressed as

CHINACOM 2010, August 25-27, Beijing, China

Copyright $\odot$ 2011 ICST 973-963-9799-97-4

DOI 10.4108/chinacom.2010.119
$s_{\mathrm{AM}}(t)=\Delta e^{j \theta} e^{j 2 \pi \Delta f_{c} t}\left(\mathcal{G}^{(r e c)}(0)+\mu_{A} x\left(t-t_{0}\right)\right)$,

where $A$ is the signal amplitude, $\theta$ is the phase, $\Delta f_{c}$ is the frequency offset, $\mathcal{G}^{(r e c)}(0)$ is the Fourier transform of the impulse response of the equivalent low-pass receive filter, $g^{(r e c)}(t)$, at zero frequency, with $\mathcal{G}^{(\text {rec })}(0)=1, \quad \mu_{A}$ is the modulation index, $x(t)=m(t) \otimes g^{(r e c)}(t)$, with $m(t)$ as the zeromean real-valued band-limited modulating signal, $t_{0}$ is the time delay, and $j=\sqrt{-1}$.

For an FSK signal, $s(t)$ is given by

$s_{\mathrm{FSK}}(t)=\Delta e^{j \theta} e^{j 2 \pi \Delta f_{c} t} \sum_{l=-\infty}^{\infty} e^{j 2 \pi f_{\Delta} s_{l}(t-l T-\varepsilon T)} g(t-l T-\varepsilon T)$,

where $f_{\Delta}$ is the frequency deviation, $T$ is the symbol period, $\varepsilon$ $(0 \leq \varepsilon \leq 1)$ is the timing offset, $g(t)$ is the overall impulse response of the transmit and receive filters, and $s_{l}$ is the symbol transmitted within the $l$ th symbol period. The data symbols $\left\{s_{l}\right\}$ are assumed to be zero-mean independent and identically distributed (i.i.d.) random variables, with values drawn from the alphabet corresponding to the $M$-FSK modulation, i.e., $s_{l} \in\left\{\tilde{s}_{m}\right.$ : $\left.\tilde{s}_{m}=2 m-1-M, m=1, \ldots, M\right\}$, with the modulation order, $M$, restricted to powers of 2 .

For an SCLD signal, $s(t)$ is written as

$s_{\mathrm{SCLD}}(t)=\Delta e^{j \theta} e^{j 2 \pi \Delta f_{c} t} \sum_{l=-\infty}^{\infty} s_{l} g(t-l T-\varepsilon T)$.

The data symbols $\left\{s_{l}\right\}$ correspond either to a PSK or QAM signal constellation, and are assumed to be i.i.d. random variables. The model in (4) also holds for CP-SCLD, with $s_{l}$ either as an information data symbol or part of the cyclic prefix (CP) [16][17]. CP-SCLD schemes employ block transmission with a CP of $L$ symbols inserted at the beginning of each block of $N$ data symbols [16]-[17]. The CP is formed by repeating the last $L$ symbols from the $N$ information data symbols $(L<N)$ of the block. To emphasize the block structure, the CP-SCLD signal model can be rewritten as [5],

$s_{\mathrm{CP}-\mathrm{SCLD}}(t)=\Delta e^{j \theta} e^{j 2 \pi \Delta f_{c} t} \sum_{b=-\infty}^{\infty} \sum_{l=0}^{N+L-1} s_{b, l} g(t-b(N+L) T-l T-\varepsilon T)$,

where $s_{b, l}$ is the symbol transmitted within the $l$ th symbol period of block $b$. Fig 1 . shows the structure of a CP-SCLD transmission block.

For the OFDM signal, $s(t)$ is expressed as

$s_{\text {OFDM }}(t)=A e^{j \theta} e^{j 2 \pi \Delta f_{c} t} \sum_{l=-\infty}^{\infty} \sum_{k=0}^{K-1} s_{k,} e^{j 2 \pi k \Delta f(t-l T-\varepsilon T)} g(t-l T-\varepsilon T)$,

where $K$ is the number of subcarriers, $s_{k, l}$ is the symbol transmitted on the $k$ th subcarrier over the lth OFDM symbol period, $\Delta f$ is the frequency separation between two adjacent 
subcarriers, $T$ is the OFDM symbol period, which equals $T_{u}+T_{c p}$, with $T_{u}=1 / \Delta f$ being the useful symbol duration and $T_{c p}$ as the CP duration. The symbols $\left\{s_{k, l}\right\}$ are assumed to be i.i.d. random variables, with values drawn either from a QAM or PSK signal constellation.

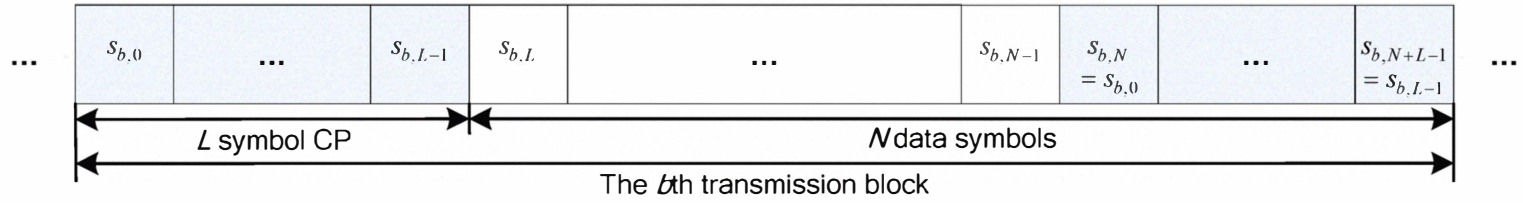

Fig. 1. CP-SCLD block structure.

The above are generic signal models. In practice, preambles, pilots, and reference symbols are often included for synchronization and estimation purposes [18]-[22]. Here, we consider a model for mobile WiMAX OFDM signals, which are specified by the IEEE 802.16e standard [18]. The reader is referred to [8] for a model of the LTE OFDM signals; this is omitted here for brevity. Fig. 2 presents the IEEE 802.16e time division duplex (TDD) frame structure, as per the current mobility certification profiles [19]-[20]. The standard frame duration can range from 2 ms to $20 \mathrm{~ms}$; however, existing WiMAX equipment supports only a $5 \mathrm{~ms}$ frame [18], [21]. The frame is divided into two subframes, one for the downlink (DL) and another for the uplink (UL). The DL-to-UL subframe ratio is variable, to support different traffic profiles.

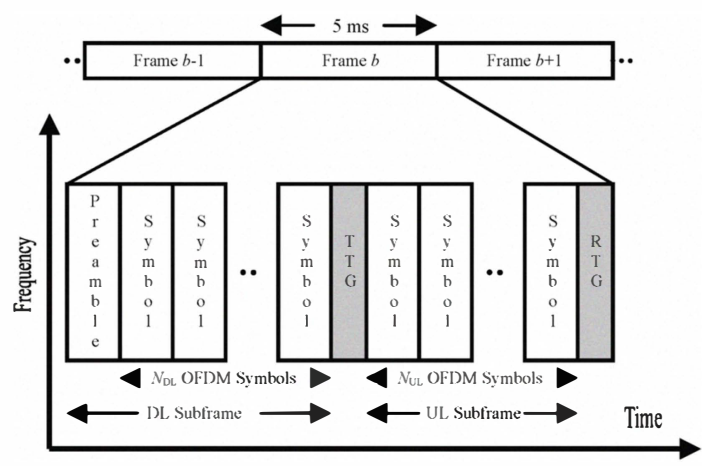

Fig. 2. TDD frame structure for mobile WiMAX.

Transition gaps separate the consecutive DL and UL subframes. In Fig. 2, TTG represents the DL-UL gap and is referred to as the transmit/receive transition gap, while RTG represents the UL-DL gap and is referred to as the receive/transmit transition gap. Note that the terminology used here follows the IEEE 802.16e standard [19]-[20]. The DL subframe starts with a preamble as the first symbol. This is used for time and frequency synchronization and uniquely identifies a serving base-station. The OFDM symbols include three types of subcarriers: the data subcarriers to transmit information, the pilot subcarriers for estimation purposes, and the null subcarriers for guard bands [19]-[20]. The pilot symbol on subcarrier $k$ is generated as $8\left(0.5-w_{k}\right) / 3$, with $w_{k}$ as a value taken from a special pseudorandom binary sequence. The preamble does not contain pilot subcarriers. For more details on the preamble structure, the reader is referred to [19]-[20].

Following the above description, a model can be written for the mobile WiMAX OFDM signal as $s(t)=s_{\mathrm{P}}(t)+s_{\mathrm{DL}}(t)+s_{\mathrm{UL}}(t)$, with
$s_{\mathrm{P}}(t), s_{\mathrm{DL}}(t)$, and $s_{\mathrm{UL}}(t)$ as the signal components corresponding to the preamble, and the DL (excluding the preamble) and UL subframes, respectively. These components are given by [7],

$$
\begin{aligned}
& s_{\mathrm{P}}(t)=\mathcal{A}_{\mathrm{P}} e^{j \theta} e^{j 2 \pi \Delta f_{c} t} \sum_{\substack{l=-\infty \\
l \bmod N_{\mathrm{F}}=0}}^{\infty} \sum_{k=-K_{\mathrm{P}} / 2, k \neq 0}^{K_{\mathrm{P}} / 2} b_{k} g\left(t-l T-l N_{\mathrm{F}}^{-1} T_{\mathrm{G}}\right) \\
& \times e^{j 2 \pi k \Delta f\left(t-l T-l N_{\mathrm{F}}^{-1} T_{\mathrm{G}}\right)}, \\
& s_{\mathrm{DL}}(t)=\mathcal{D}_{\mathrm{DL}} e^{j \theta} e^{j 2 \pi \Delta f_{c} t} \sum_{\substack{l=-\infty \\
l \bmod N_{\mathrm{F}} \leq N_{\mathrm{DL}} \\
l \bmod N_{\mathrm{F}} \neq 0}}^{\infty} \sum_{k=-K_{\mathrm{DL}} / 2, k \neq 0}^{K_{\mathrm{DL}} / 2} c_{k, l} g\left(t-l T-\left\lfloor l N_{\mathrm{F}}^{-1}\right\rfloor T_{\mathrm{G}}\right) \\
& \times e^{j 2 \pi k \Delta f\left(t-l T-\left[l N_{\mathrm{F}}^{-1} \mid T_{\mathrm{G}}\right)\right.}, \\
& s_{\mathrm{UL}}(t)=A_{\mathrm{L}} e^{j \theta} e^{j 2 \pi \Delta f_{c} t} \sum_{\substack{l=-\infty \\
l \bmod N_{\mathrm{F}}>N_{\mathrm{DL}}}}^{\infty} \sum_{\substack{k=-K_{\mathrm{UL}} / 2, k \neq 0 \\
K_{\mathrm{UL}} / 2}}^{\infty} d_{k, l} g\left(t-l T-\left\lfloor l N_{\mathrm{F}}^{-1}\right\rfloor T_{\mathrm{G}}-T_{\mathrm{TG}}\right) \\
& \times e^{j 2 \pi k \Delta f\left(t-l T-\left\lfloor l N_{\mathrm{F}}^{-1}\right\rfloor T_{\mathrm{G}}-T_{\mathrm{TG}}\right)},
\end{aligned}
$$

where $K_{\mathrm{P}}, K_{\mathrm{DL}}$, and $K_{\mathrm{UL}}$ are the number of non-null subcarniers in the preamble, DL, and UL symbols, respectively, $\mathcal{A}_{\mathrm{p}}, \mathcal{A}_{\mathrm{bL}}$, and $\mathcal{A}_{\mathrm{UL}}$ are the amplitude factors equal to $1 / \sqrt{K_{\mathrm{P}}}, 1 / \sqrt{K_{\mathrm{DL}}}$, and $1 / \sqrt{K_{\mathrm{UL}}}$, respectively, $b_{k}$ is the data symbol transmitted on the $k$ th subcarrier of the preamble, $c_{k, l}$ and $d_{k, l}$ are the (data and pilot) symbols transmitted on the $k$ th subcarrier and within the $l$ th OFDM symbol of the DL and UL subframes, respectively, $N_{\mathrm{F}}$ is the number of symbols in the frame, $N_{\mathrm{DL}}$ and $N_{\mathrm{UL}}$ are the number of symbols in the DL (excluding the preamble) and UL subframes, respectively, $T_{\mathrm{G}}=T_{\mathrm{TG}}+T_{\mathrm{RG}}$ is the total duration of the transition gaps within each frame, with $T_{\mathrm{TG}}$ and $T_{\mathrm{RG}}$ as the TTG and RTG transition gaps, respectively, and $\lfloor$.$\rfloor denotes the floor function. Note that the data$ symbols are assumed to be zero-mean i.i.d. random variables, with values taken from either a PSK or QAM signal constellation.

Note that $s_{\mathrm{P}}(t)$ in (7.a) depends on the position of the preamble at the beginning of each frame. The preamble index, $l$, is an integer multiple of the number of OFDM symbols in a frame $\left(l \bmod N_{\mathrm{F}}=0\right)$. Furthermore, the need to consider the total duration of the transition gaps within each frame contributes the shift of $g(t)$ and $e^{j 2 \pi k \Delta f}$ by $l N_{\mathrm{F}}^{-1} T_{\mathrm{G}}$, where $l N_{\mathrm{F}}^{-1}$ provides the frame index. Note that the symbol on each subcarrier, $k$, is the same for all preambles ( $b_{k}$ does not depend on $l$ ).

\section{Signal CyClostationarity}

The time domain cyclostationarity parameters of the signals of interest are summarized in the following; for the definitions of these parameters the reader is referred to Appendix A. 


\section{A. First-Order Signal Cyclostationarity}

The first-order cyclic cumulant (CC) of the AM signals and the cycle frequency $(\mathrm{CF})$ are given respectively as [1]

$c_{r}(\beta)_{1,0}=\mathcal{A} e^{j \theta}$,

$\kappa_{r, 1,0}=\left\{\beta: \beta=\Delta f_{c}\right\}$.

Note that the CC equals zero at frequencies other than CF; this is valid for all subsequent results. The $\mathrm{CC}$ depends only on the signal amplitude and phase, while the CF depends only on the frequency offset. The CC magnitude, $\left|c_{r}(\beta)_{1,0}\right|=\mathcal{A}$, depends only on the signal amplitude.

For $f_{\Delta}=i T^{-1}$, with $i$ as an integer, the FSK signals exhibit first-order cyclostationarity, with the first-order CCs and the set of CFs given respectively as [1]

$c_{r}(\beta)_{1,0}=A M^{-1} e^{j \theta} e^{-j 2 \pi \kappa \%}$,

$\kappa_{r, 1,0}=\left\{\beta: \beta=\gamma+\Delta f\right.$, with $\gamma=p T^{-1}$ and $\left.p= \pm i, \ldots, \pm(M-1) i\right\}$.

The first-order CCs depend on the modulation order, signal amplitude, phase, timing offset, and product $f_{\Delta} T$ and alphabet (both through $\gamma T=p$ ). The CC magnitude, $\left|c_{r}(\beta)_{1,0}\right|=A, M^{-1}$, depends only on the modulation order and signal amplitude. Moreover, this decreases with increasing $M$. In addition, according to (11), the number of first-order CFs is equal to the modulation order, $M$, and for any given $M$, the CFs depend on the frequency offset, and frequency deviation and alphabet (both through $\gamma$ ). Also from (11), it can be easily seen that the distance between any two adjacent CFs equals $2 f_{\Delta}$.

Based on the symmetry of the points in the signal constellation, one can infer that SCLD, CP-SCLD, and OFDM signals do not exhibit first-order cyclostationarity. The same holds for the noise, as this is a zero-mean stationary process. The first-order cyclostationarity can be exploited to distinguish between AM (characterized by a single first-order CF), $M$-FSK ( $M$ first-order CFs), and SCLD, CP-SCLD, OFDM, and noise (no first-order CFs).

\section{B. Second-Order Signal Cyclostationarity}

The second-order/ one-conjugate CCs and the set of CFs for the SCLD signals are given respectively as [2]-[3]

$c_{r}(\beta ; \tau)_{2,1}=\mathrm{v}(\tau) T^{-1} e^{-j 2 \pi \times \beta T} \Lambda(\tau)+c_{w}(\beta ; \tau)_{2,1}$,

$\kappa_{r, 2,1}=\left\{\beta: \beta=i T^{-1}, i\right.$ integer $\}$,

where $u(\tau)=\mathcal{A}^{2} e^{-j 2 \pi \Delta f_{c} \tau} c_{s, 2,1}$ and $\Lambda(\tau)=\int_{-\infty}^{\infty} g(t) g^{*}(t+\tau) e^{-j 2 \pi \beta t} d t$.

We refer to this as to the symbol-induced cyclostationarity. Note that the CC expression in (12) is valid only at the CFs given in (13), and for certain delays having values around zero (the range of these delays depends on the pulse shape). From (12), it is apparent that the $\mathrm{CC}$ magnitude for the signal component reaches a maximum at zero delay.

The second-order/ one-conjugate CCs and set of CFs for the CPSCLD signals are respectively given as [5]

$c_{r}(\beta ; \tau)_{2,1}=$

$\cup(\tau) T^{-1} e^{-j 2 \pi \beta \beta T} \Lambda(\tau)+c_{w}(\beta ; \tau)_{2,1}$, for delays around $\tau=0$ and $\beta=i T^{-1}, i$ integer,

$v(\tau)[(N+L) T]^{-1} e^{-j 2 \pi \beta \beta} \int_{-\infty}^{\infty} \sum_{l=0}^{L-1} g(t-l T) g^{*}(t-l T \mp N T+\tau) e^{-j 2 \pi \beta t} d t+c_{w}(\beta ; \tau)_{2,1}$,

for delays around $\tau= \pm N T$ and $\beta=b[(N+L) T]^{-1}, b$ integer, $\kappa_{r, 2,1}=\left\{\beta: \beta=i T^{-1}, i\right.$ integer, when $\tau$ varies around zero $\}$

$\cup\left\{\beta: \beta=b[(N+L) T]^{-1}, b\right.$ integer, when $\tau$ varies around $\left.\pm N T\right\}$.

These results reflect the similarities and differences between CPSCLD and SCLD signals. As such, the second-order/ one-conjugate CCs and set of CFs is as for SCLD around zero delay. On the other hand, non-zero CCs are also obtained for CP-SCLD for delays around $\pm N T$, which accounts for the existence of the CP (this is referred to as the CP-induced cyclostationarity). The periodicity of the time-varying cumulant is related to the symbol period in the former case and the block duration in the latter case; this is reflected in the expressions of the CFs.

The second-order/ one-conjugate CCs and the set of CFs for OFDM signals are respectively given as [2], [9]

$c_{r}(\beta ; \tau)_{2,1}=\mathcal{A}^{2} c_{s, 2,1} T^{-1} e^{-j 2 \pi \varepsilon \beta T} \Xi_{K}(\tau) \Lambda(\tau)+c_{w}(\beta ; \tau)_{2,1}$,

$\kappa_{r, 2,1}=\left\{\beta: \beta=i T^{-1}, i\right.$ integer $\}$,

where $\Xi_{K}(\tau)=e^{j \pi(K-1) \Delta f \tau} \sin (\pi K \Delta f \tau) / \sin (\pi K \Delta f \tau)$. As for the SCLD and CP-SCLD signals, the expression for the second-order/ oneconjugate CCs in (16) is valid only at CFs given in (17) and for certain delays. It is noteworthy that the values of such delays are around zero and $\pm T_{u}$, with the latter due to the existence of the CP.

Similar to generic OFDM signals, the mobile WIMAX OFDM signals also exhibit second-order cyclostationarity with nonzero CCs at delays around zero and $\pm T_{u}$ (symbol- and CP-induced cyclostationarity, respectively). There is also preamble- and pilotinduced cyclostationarity. The preamble-induced cyclostationarity is due to the preamble repetition in each frame [7]. This results in the second-order/ one-conjugate CCs at CFs and delays equal to integer multiples of the frame duration, $T_{F}$, with the set of CFs respectively given by [7]

$$
\begin{aligned}
& c_{r}(\beta ; \tau)_{2,1}=T_{\mathrm{F}}^{-1} \sigma_{b}^{2} \int_{-\infty}^{\infty}|g(t)|^{2} e^{-j 2 \pi \beta t} d t, \\
& \kappa_{r, 2,1}=\left\{\beta: \beta=i T_{\mathrm{F}}^{-1}, i \text { integer }\right\},
\end{aligned}
$$

where $\sigma_{b}^{2}$ is the variance of the data symbols in the preamble.

The second-order cyclostationarity can be used to distinguish between SCLD (symbol-induced), CP-SCLD (symbol- and CPinduced), and OFDM (CP-induced) signals, as well as to identify standard OFDM signals, such as mobile WiMAX (symbol-, CP- and preamble-induced).

\section{Higher-Order Signal Cyclostationarity}

The $n$ th-order/ q-conjugate CCs and the set of CFs for the SCLD signals are given respectively as [3]

$$
\begin{aligned}
& c_{r}(\beta ; \tau)_{n, q}=\mathcal{A}^{n} c_{s, n, q} T^{-1} e^{-j 2 \pi \approx \gamma T} e^{j(n-2 q) \theta} e^{j 2 \pi \Delta f_{c} \sum_{u=1}^{n-1}()_{u} \tau_{u}} \\
& \times \int_{-\infty}^{\infty} p^{(*)_{n}}(t) \prod_{u=1}^{n-1} p^{\left(*_{u}\right.}\left(t+\tau_{u}\right) e^{-j 2 \pi t} d t, \\
& \kappa_{r, n, q}=\left\{\beta: \beta=\gamma+(n-2 q) \Delta f, \gamma=i T^{-1}, i \text { integer }\right\},
\end{aligned}
$$

where $\tau=\left[\tau_{1} \ldots \tau_{n-1}\right]^{\dagger}$, with $\dagger$ as the transpose, $c_{s, n, q}$ is the $n$ thorder/q-conjugate cumulant of the signal constellation, $\left({ }^{*}\right)_{u}$ represents a possible conjugation of the $u$ th term so that the total number of conjugations is $q, u=1, \ldots, n$, and $(-)_{u}$ is the minus sign from the conjugation $\left({ }^{*}\right)_{u}, u=1, \ldots, n-1$. Examples of $c_{s, n, q}$ values for diverse signal constellations are provided in Table 1; for other signal constellations the reader is referred to [3]-[4]. As $w(t)$ is a 
stationary, zero-mean Gaussian process, its cumulants are time independent, and non-zero only for the second-order. Since higherorder $(n \geq 3)$ CCs are of interest, the noise contribution does not appear in (20). Note that (20) holds only for a certain delay range and CFs given in (21); otherwise, this is zero. From (20), it follows that the timing, phase, and frequency offsets yield a CC rotation. Moreover, the CC is directly proportional to the $n$th power of the signal amplitude, $n$ th-order/ $q$-conjugate cumulant of the signal constellation, reciprocal of the symbol period, and functional $\Xi(n, q, \tau, \gamma, p(t))=\int_{-\infty}^{\infty} p^{\left(*_{n}\right.}(t) \prod_{u=1}^{n-1} p^{\left(*_{u}\right.}\left(t+\tau_{u}\right)^{-j 2 \pi t u} d t$. This functional depends on the CC order, number of conjugations, delay-vector, frequency $\gamma$, and pulse shape.

The (symbol-induced) higher-order cyclostationarity can be used to discriminate SCLD signals, i.e., to identify $M$-ASK, $M$-PSK, and $M$-QAM signals based on the values in Table 1 . For example, the recognition of 8-PSK and 16-PSK signals necessitates CCs of order eight (see the columns in the table corresponding to these modulations).

Table 1. Theoretical cumulants of unit signal constellations [3]-[4].

\begin{tabular}{|c|c|c|c|c|c|c|}
\hline & BPSK & QPSK & 8 -PSK & 16-PSK & 16-QAM & $64-$ QAM \\
\hline$c_{s, 2,0}$ & 1 & 0 & 0 & 0 & 0 & 0 \\
\hline$c_{s, 2,1}$ & 1 & 1 & 1 & 1 & 1 & 1 \\
\hline$c_{s, 4,0}$ & -2 & 1 & 0 & 0 & -0.68 & -0.619 \\
\hline$c_{s, 4,1}$ & -2 & 0 & 0 & 0 & 0 & 0 \\
\hline$c_{s, 4,2}$ & -2 & -1 & -1 & -1 & -0.68 & -0.619 \\
\hline$c_{s, 6,0}$ & 16 & 0 & 0 & 0 & 0 & 0 \\
\hline$c_{s, 6,1}$ & 16 & -4 & 0 & 0 & 2.08 & 1.7972 \\
\hline$c_{s, 6,2}$ & 16 & 0 & 0 & 0 & 0 & 0 \\
\hline$c_{s, 6,3}$ & 16 & 4 & 4 & 4 & 2.08 & 1.7972 \\
\hline$c_{s, 8,0}$ & -272 & -34 & 1 & 0 & -13.9808 & -11.5022 \\
\hline$c_{s, 8,1}$ & -272 & 0 & 0 & 0 & 0 & 0 \\
\hline$c_{s, 8,2}$ & -272 & 34 & 0 & 0 & -13.9808 & -11.5022 \\
\hline$c_{s, 8,3}$ & -272 & 0 & 0 & 0 & 0 & 0 \\
\hline$c_{s, 8,4}$ & -272 & -34 & -33 & -33 & -13.9808 & -11.5022 \\
\hline
\end{tabular}

\section{Signal CyClostationarity - APPlications}

A. First-, Second-, and Higher-Order CCs for AM, FSK, SCLD, CPSCLD, and OFDM Signal Detection and Classification

The theoretical results presented in Section III yield several important conclusions: - First-order cyclostationarity can be used to discriminate AM (a single $\mathrm{CF}$ ), $M$-FSK ( $M \mathrm{CFs}$ ), and SCLD, CP-SCLD, OFDM, and noise (no CFs); - Second-order cyclostationarity can be employed to distinguish between SCLD (symbol-induced cyclostationarity), CP-SCLD (both symbol- and CP-induced cyclostationarity), OFDM(CP-induced cyclostationarity), and noise (neither symbol- nor CP-induced cyclostationarity). Moreover, standard signals can be identified. Here we consider the example of mobile WiMAX OFDM signals (symbol-, CP-, and preamble-induced cyclostationarity); for the LTE OFDM signals the reader is referred to [8] and [9]; - Furthermore, higher-order cyclostationarity can be employed to identify the modulation type of SCLD and CP-SCLD signals, i.e., $M$-ASK, $M$-PSK, or $M$ QAM. The application of signal cyclostationarity to signal detection and classification is illustrated in Fig. 3. This provides a general algorithm; in specific frequency bands only some of the considered signals are expected, and the algorithm can be simplified accordingly.

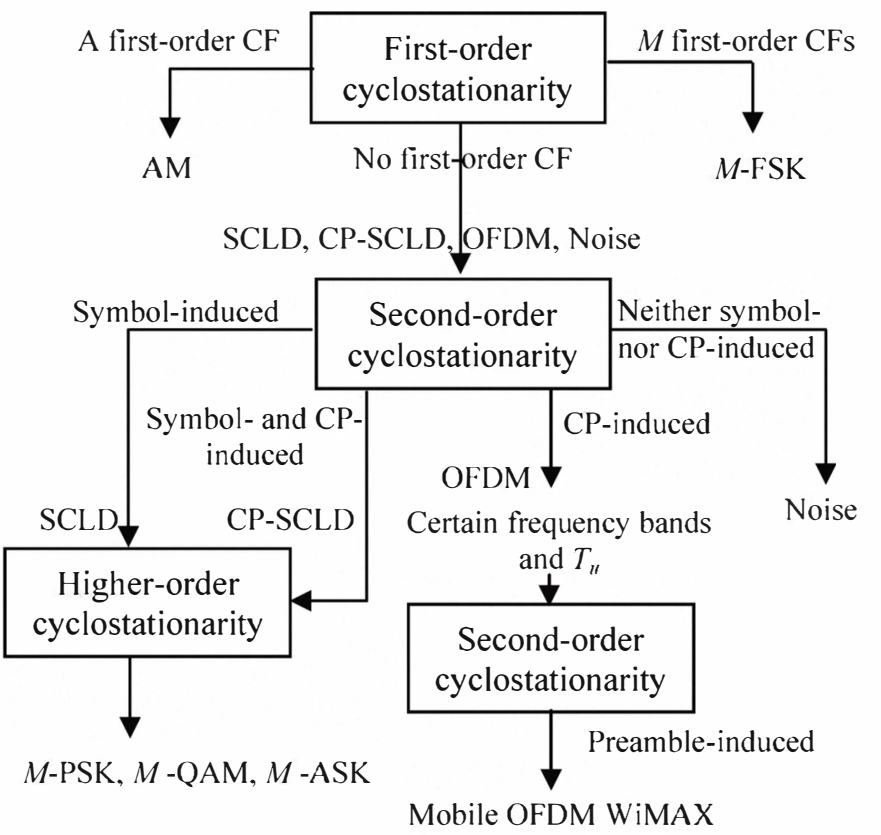

Fig. 3. Application of cyclostationarity to signal detection and classification.

Due to space constraints, we provide only a brief treatment on the application of second-order cyclostationarity for signal detection and classification. We first distinguish between the SCLD/noise and the CP-SCLD/OFDM classes by exploiting the CP-induced features. OFDM and CP-SCLD signals are associated with peaks in the second-order/ one-conjugate CC magnitude at zero CF and delays around $\pm T_{u}$ and $\pm N T$, respectively. No such peaks exist for SCLD signals and noise. Note that the second-order/ one conjugate CC magnitude of the received signal is estimated at zero CF $(\beta=0)$ and over a delay range large enough to cover possible values of the $T_{u}$ and $N T$. The minimum delay must be sufficiently far from zero to serve as an unambiguous discriminating feature. Over the considered delay range, we select the delay value for which the CC magnitude reaches a local maximum. The cyclostationarity test developed in [23] is used to check whether $\beta=0$ is indeed a CF for the selected delay. It involves the comparison of a statistic calculated from the estimated secondorder/ one-conjugate $\mathrm{CC}$ at the aforementioned $\mathrm{CF}$ and delay, against a threshold. A more detailed description is given in 
Appendix B. If $\beta=0$ is found to be a CF, i.e., the test statistic exceeds the threshold, the decision is CP-SCLD/OFDM class; otherwise SCLD/noise class. After signal class identification, SCLD signals and noise, and CP-SCLD and OFDM signals are respectively distinguished by exploiting the symbol-induced second-order cyclostationarity, viz., the existence of peaks in the second-order/ one-conjugate CC magnitude of SCLD and CPSCLD signals at non-zero $\mathrm{CF}$ and zero delay. As such, the magnitude of the second-order/ one-conjugate $\mathrm{CC}$ is estimated at zero delay and over a $\mathrm{CF}$ range which excludes zero $\mathrm{CF}$. The frequency for which the $\mathrm{CC}$ magnitude reaches a local maximum is selected as a candidate CF. The same cyclostationarity test is then employed to check whether the candidate CF is indeed a CF for zero delay. In this case, the statistic is calculated based on the estimated second-order/ one-conjugate $\mathrm{CC}$ at candidate $\mathrm{CF}$ and zero delay. If the candidate $\mathrm{CF}$ is found to be a $\mathrm{CF}$, the decision that an SCLD or an CP-SCLD signal is present is made.

Furthermore, for the recognition of the mobile WiMAX OFDM signal in the frequency bands specified by the standard [19]-[21], the estimated delay related to the CP-induced cyclostationarity [12], is compared against those corresponding to the standard [9], and the existence of the preamble-induced second-order cyclostationarity verified. For the latter, the second-order/ oneconjugate $\mathrm{CC}$ is estimated at zero $\mathrm{CF}$ and delay equal to $T_{F}$, and the previously mentioned cyclostationarity test is applied to make a decision on the existence of the WiMAX OFDM signal.

Note that second-order CCs at non-zero CFs can be additionally employed to verify the CP-induced cyclostationarity of OFDM and CP-SCLD signals; this improves detection and classification performance at the cost of increased complexity [2]. Also, more than one CF can be checked when exploiting the preamble-induced cyclostationarity for mobile OFDM WiMAX signal recognition [7]; in this case, an extension of the previously mentioned cyclostationarity test needs to be applied [24].

For approaches based on first- and higher-order cyclostationarity, the reader is referred to [1], [3], [4]. Moreover, the exploitation of spatial receive diversity to improve the classification performance is addressed in [3].

Note that the first- and second-order cyclostationarity based signal detection and classification algorithms have the advantage of avoiding the need for the recovery of the carrier, waveform, and symbol timing, or the estimation of noise and signal power [1]-[2], [5]-[9]. On the other hand, the higher-order cyclostationarity-based algorithm requires estimation of the signal amplitude and waveform recovery [3].

\section{B. First- and Second-Order CCs for Blind Parameter Estimation}

In addition to distinctive features for signal detection and classification, the $\mathrm{CF}$ domain gives information on diverse signal parameters. Based on results presented in Section III, we can draw the following conclusions: - According to (9), the frequency offset can be estimated for AM signals from the position of the CF; Based on (11), the frequency deviation of FSK signals can be obtained from the distance between two consecutive CFs, after which the frequency offset can be calculated based on the position of a specific CF; - From (13) and (15), it can be seen that the symbol-induced second-order cyclostationarity exhibited by SCLD and CP-SCLD signals yields information on the signal period ${ }^{1}$; - Further, the CP-induced second-order cyclostationarity of CPSCLD signals yields estimates of the number of data and CP symbols (see (14) and (15) and the comments after); - The CPinduced second-order cyclostationarity of OFDM signals provides estimates of the useful symbol and CP durations (see (16) and (17) and the comments which follow them).

We present an example of the application of second-order cyclostationarity to blind parameter estimation, viz., the estimation of the number of data and CP symbols in a CP-SCLD transmission block. Both symbol- and CP-induced cyclostationarity are used in a three stage process: (1) The symbol period is estimated from the corresponding symbol-induced peak in the second-order/ oneconjugate CC magnitude at zero delay; (2) The number of data symbols in a block, $N$, is estimated by exploiting the existence of the CP-induced peak in the CC magnitude at delay $N T$ and zero CF; (3) The number of CP symbols, $L$, is estimated based on the CP-induced peaks in the CC magnitude at delay $N T$ and CFs other than zero. A perfect estimate of the symbol period is considered here, and, thus, we focus only on the second and third steps. For the second step, the CC magnitude of the received signal is estimated at $\beta=0$ and a delay range whose limits are set using $a$ priori knowledge of the minimum and maximum values of $N$. The delay for which the $\mathrm{CC}$ magnitude reaches a local maximum is selected, i.e., $\hat{\tau}=\operatorname{argmax}\left\{\left|\hat{c}_{r}(0 ; \tau)\right|\right\}, \quad \tau \in\left[N_{\min } T, N_{\max } T\right]$, where $N_{\min }$ and $N_{\max }$ as the $e^{\tau}$ minimum and maximum numbers of data symbols in a transmission block, respectively, and the hat symbol standing for the estimated value. Note that this information may be available from the classification algorithm. The number of data symbols in a block is chosen as $\hat{N}=\lfloor\hat{\tau} / T\rceil$, with $\lfloor$.$\rceil denoting the$ nearest integer function. The $\mathrm{CC}$ magnitude is then estimated at the previously selected delay and for a certain range of positive CFs. Given that this range should be small to limit the number of estimates and that significant CC magnitude values are attained only for a small number of CFs greater than zero, the value of the integer $b$ can be limited to an appropriately chosen $b_{\max }$. Moreover, as the CFs in the considered range are given by $b[(N+L) T]^{-1}$, with $b$ as a positive integer and $L$ unknown, we choose the $C F$ range by taking into account that $0<L<N$. The frequency for which the $\mathrm{CC}$ magnitude reaches a local maximum, $\hat{\beta}$, is selected as $\hat{\beta}=\operatorname{argmax}\left\{\left|\hat{c}_{r}(\beta ; \hat{\tau})\right|\right\}, \beta \in\left((2 \hat{N} T)^{-1}, b_{\max }(\hat{N} T)^{-1}\right), \quad$ with $\hat{N}$ previously obtained. Then, the number of symbols in the CP is estimated as $\hat{L}=\left\lfloor b(\hat{\beta} T)^{-1}-\hat{N}\right\rceil$, with $b$ as the minimum integer between 1 and $b_{\max }$ for which a positive value is obtained for $\hat{L}$. Note that, although theory indicates that the maximum peak occurs for $b=1$, the possibility of selecting another peak under short observation period and low signal-to-noise ratio (SNR) conditions justifies the constraint of $b \in\left[1, b_{\max }\right]$. Note that the

\footnotetext{
${ }^{1}$ Note that in practice we use discrete-time signals obtained by sampling the corresponding continuous-time signals at rate $f_{s}$. Hence, the CCs and CFs have to be correspondingly considered [1]-[9]. For example, the CFs $i T^{-1}$ become $i\left(T f_{s}\right)^{-1}$. Since $f_{s}$ is a known parameter, $T$ can be obtained from these CFs.
} 
second-order cyclostationarity-based parameter estimation algorithm has the important advantage of avoiding the need for carrier and waveform recovery, or estimation of noise and signal power [6]. On the other hand, the frequency offset represents a parameter which is estimated from the first-order cyclostationarity.

\section{SimUlation Results}

The application of second-order signal cyclostationarity to signal detection and classification, and blind parameter estimation was investigated using simulation experiments.

\section{A. Simulation Setup}

CP-SCLD signals are simulated with a 16-QAM constellation, bandwidth $B=1.25 \mathrm{MHz}$, and a root raised cosine pulse shape with a roll-off factor of 0.35 at the transmit side. Unless otherwise indicated, $L=32(L / N=1 / 4)$. In addition, $f_{s}$ is set to $5 \mathrm{MHz}$, $\Delta f_{c}$ to $500 \mathrm{kHz}, \theta$ and $\varepsilon$ are random variables uniformly distributed over $[-\pi, \pi)$ and $[0,1)$, respectively, and $b_{\max }$ equals 8 . Additive white Gaussian noise (AWGN), and ITU-R pedestrian and vehicular A fading channels are considered. The delay spread profile of the fading channels is specified in [25]. The maximum Doppler spread is $9.72 \mathrm{~Hz}$ and $194.44 \mathrm{~Hz}$ for the pedestrian and vehicular fading channels, respectively. The out-of-band noise is removed at the receive side, and the SNR is set at the output of the receive filter. The decision threshold with the cyclostationarity test set to 27.631. Unless otherwise indicated, the observation periods equal $6.4 \mathrm{~ms}$ and $12.8 \mathrm{~ms}$, respectively. The probability of correct signal detection and classification, $P_{d c}^{(s s)}$, with $s$ as the signal type, is used for performance evaluation. We assume perfect knowledge of the signal bandwidth; the reader is referred to [2] for the effect of bandwidth estimation on performance. The normalized mean squared error (NMSE) is used as the performance measure for parameter estimation. Note that normalization is performed with respect to the second power of the true parameter value.

\section{B. Performance of Cyclostationarity-Based Algorithms}

The detection and classification performance for the CP-SCLD signals, and the performance of the estimators for $\hat{N}$ and $\hat{L}$ are respectively plotted versus SNR in Fig. 4 and 5. Additional results are given in [1]-[9]. The probability $P_{d c}^{\text {(CP-SCLD|CP-SCLD) }}$ is plotted versus SNR in Fig. 4 a). The performance is very similar for the AWGN and pedestrian fading channels. This behaviour holds regardless of the observation period. Neither $6.4 \mathrm{~ms}$ nor $12.8 \mathrm{~ms}$ is adequate to provide an acceptable performance in the vehicular fading channel, even with an increase in the SNR and that a longer observation period is needed (see Fig. 4 b)).

Figs. 5 a) and b) show the performance dependence of the estimators for the number of data symbols, $\hat{N}$, and CP symbols, $\hat{L}$, in a CP-SCLD transmission block, respectively. The NMSE of the corresponding parameter estimator is plotted as a function of SNR, for the three channels. Interestingly, the channel type has little effect on the performance for either estimator. As expected, a longer observation period leads to an improved performance. The estimator for the number of data symbols performs better than that for the CP symbols; this can be easily explained, as the latter is dependent on the former

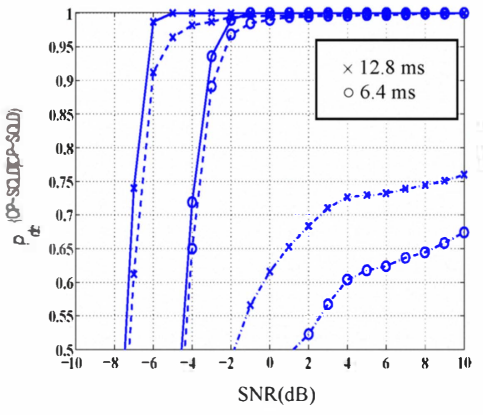

a)

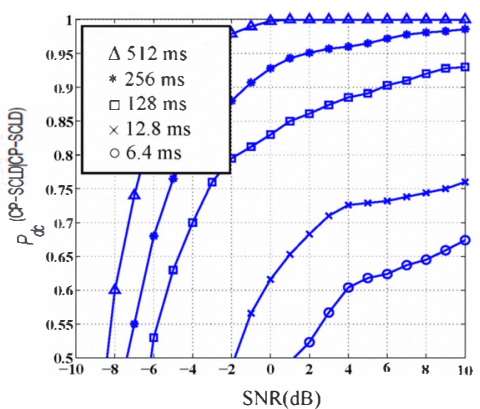

b)
Fig. 4. Performance for detection and classification of the CPSCLD signals versus SNR in AWGN (solid line), ITU-R pedestrian A (dashed line), and ITU-R vehicular A (dash-dot line) channels.

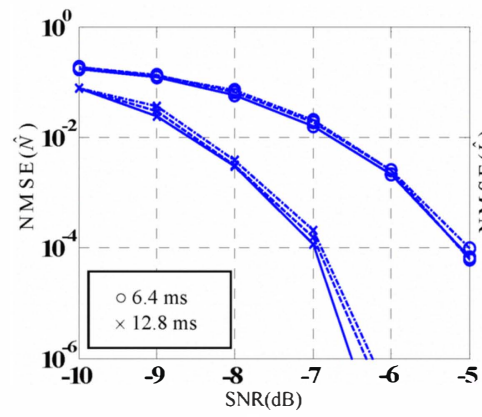

a)

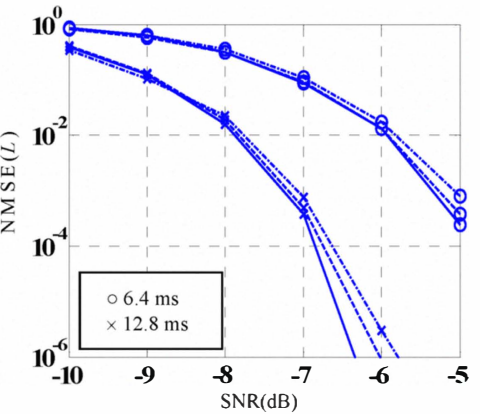

b)
Fig. 5. Performance of a) $\hat{N}$ and b) $\hat{L}$ versus SNR in AWGN (solid line), ITU-R pedestrian A (dashed line), and ITU-R vehicular A (dash-dot line) channels.

Note that the performance of both detection and classification and parameter estimation algorithms depends on the ratio of $L$ and $N$. For example, if $L=N / 8$, a performance degradation is observed relative to the $L=N / 4$ case [5]-[6]. The weakened CP-induced peak in the $\mathrm{CC}$ magnitude resulting from the shorter $\mathrm{CP}$ duration can be more easily missed in the search. Note that the performance can be improved by using a larger observation period [5]-[6].

\section{Conclusion}

An overview of the work carried out by our group on the application of cyclostationarity to joint signal detection, classification, and blind parameter estimation has been presented. First-order cyclostationarity is used to identify AM versus $M$-FSK versus SCLD/CP-SCLD/OFDM/noise classes, second-order cyclostationarity is utilized to classify SCLD versus CP-SCLD versus OFDM versus noise, and higher-order cyclostationarity is employed to identify the modulation type of SCLD and CPSCLD signals. Moreover, second-order cyclostationarity can be used to identify standard OFDM signals, such as mobile WiMAX. First- and second-order cyclostationarity can be also applied for blind parameter estimation, such as frequency offset, frequency deviation, useful symbol duration, symbol period, and number of data and CP symbols in a transmission block. Further work is in progress to address additional standard signals and multiple antenna scenarios. 


\section{ACKNOWLEDGMENT}

This work has been supported in part by the Natural Sciences and Engineering Research Council of Canada and Defence Research and Development Canada. The authors thank the other members of the team for their contributions.

\section{REFERENCES}

[1] O. A. Dobre, S. Rajan, and R. Inkol, "Joint signal detection and classification based on first order cyclostationarity for cognitive radios," EURASIP Journal on Advances in Signal Processing, ID 656719, 2009, DOI:10.1155/2009/656719.

[2] A. Punchihewa, Q. Zhang, O. A. Dobre, C. Spooner, S. Rajan, and R. Inkol, "On the cyclostationarity of OFDM and single carrier linearly digitally modulated signals in time dispersive channels: Theoretical developments and application," IEEE Transactions on Wireless Communications, accepted for publication.

[3] O. A. Dobre, A. Abdi, Y. Bar-Ness, and W. Su, "Cyclostationaritybased modulation classification of linear digital modulations in flat fading channels," Kluwer Wireless Personal Communications Journal, July 2009, DOI: 10.1007/ s1 1277-009-9776-2.

[4] O. A. Dobre, A. Abdi, Y. Bar-Ness, and W. Su, "A survey of automatic modulation classification techniques: classical approaches and new trends," IET Commun., vol. 1, pp. 137-156, April 2007.

[5] Q. Zhang, O. A. Dobre, S. Rajan, R. Inkol, and E. Serpedin, "Cyclostationarity approach for the recognition of cyclically prefixed single carrier signals in cognitive radio," in Proc. IEEE ICC, 2010.

[6] Q. Zhang, O. A. Dobre, S. Rajan, and R. Inkol, "Cyclostationarity approach to joint blind estimation of CP-SCLD block transmission parameters for cognitive radio," in Proc. IEEE DySPAN, 2010.

[7] A. Al-Habashna, O. A. Dobre, R. Venkatesan, and D. Popescu, "WiMAX signal detection algorithm based on preamble-induced second-order cyclostationarity," submitted to IEEE GLOBECOM 2010.

[8] A. Al-Habashna, O. A. Dobre, R. Venkatesan, and D. Popescu, "Cyclostationarity-based detection of LTE OFDM signals for cognitive radio systems," submitted to IEEE GLOBECOM 2010.

[9] A. Al-Habashna, O. A. Dobre, R. Venkatesan, and D. Popescu, "Joint signal detection and classification of mobile WiMAX and LTE OFDM signals for cognitive radio," submitted to VTC Fall 2010.

[10] H. Bolcskei, "Blind estimation of symbol timing and carrier frequency offset in wireless OFDM systems," IEEE Trans. Commun., vol. 49, pp. 988-999, 2001.

[11] M. Shi, Y. Bar-Ness, and W. Su, "Blind OFDM systems parameters estimation for software defined radio," in Proc. IEEE DySPAN, 2007, pp. 119-122.

[12] A. Bouzegzi, P. Jallon, and P. Ciblat, "A second order statistics based algorithm for blind recognition of OFDM based systems," in Proc. IEEE GLOBECOM, 2008, pp. 1-5.

[13] K. Kim, I. A. Akbar, K. K. Bae, J. Urn, C. M. Spooner, and J. H. Reed, "Cyclostationary approaches to signal detection and classification in cognitive radio," in Proc. IEEE DySPAN, 2007, pp. 212-215.

[14] V. Le Nir, T. van Waterschoot, M. Moonen, and J. Duplicy, "Blind CP-OFDM and ZP-OFDM parameter estimation in frequency selective channels," EURASIP Journal on Wireless Communications and Networking, ID 315765, 2009, DOI: 10.1155/2009/3157652009.

[15] M. Oner and F. Jondral, "On the extraction of the channel allocation information in spectrum pooling system," IEEE Trans. Sele. Areas in Commun., vol. 25, pp. 558-565, 2007.

[16] D. Falconer, S. L. Ariyavisitakul, A. Benyamin-Seeya, and B. Eidson, "Frequency domain equalization for single carrier broadband wireless systems," IEEE Commun. Mag., vol. 40, pp. 58-66, Apr. 2002.

[17] B. Devillers, J. Louveaux, and L. Vandendorpe, "Exploiting cyclic prefix for performance improvement in single carrier systems," in Proc. SPAWC, 2006, pp. 1-5.

[18] WiMAX forum, WiMAX Forum Mobile System Profile, 2007.

[19] IEEE Std. 802.16, Part 16: Air Interface for Fixed Broadband Wireless Access Systems, 2004.

[20] IEEE Std. 802.16, Part 16: Air Interface for Fixed and Mobile Broadband Wireless Access Systems, Amendment 2: Physical and medium Access Control Layers for Combined Fixed and Mobile Operations in License Bands and Corrigendum 1, 2005.

[21] J. G. Andrews, A. Ghosh, and R. Muhamed, Fundamentals of WiMAX: Understanding Broadband Wireless Networking. PrenticeHall, 2007.

[22] 3GPP TS 36.211: Evolved Universal Terrestrial Radio Access (E-UTRA); Physical channels and modulation.

[23] A. V. Dandawate and G. B. Giannakis, "Statistical test for presence of cyclostationarity," IEEE Trans. Sig. Proc., vol. 42, pp. 2355-2369, 1994.

[24] J. Lunden et al., "Spectrum sensing in cognitive radios based on multiple cyclic frequencies," in Proc. IEEE CROWNCOM, 2007, pp. 37-43.

[25] A. F. Molisch, Wireless Communications, Wiley-IEEE Press, New York, NY, USA, 2005.

[26] C. M. Spooner and W. A. Gardner, "The cumulant theory of cyclostationary time-series, part I: foundation and part II: development and applications," IEEE Trans. Sig. Proc., vol. 42, pp. 3387-3429, Dec. 1994.

[27] A. V. Dandawate and G. B. Giannakis, "Asymptotic theory of mixed time averages and kth-order cyclic-moment and cumulant statistics," IEEE Trans. Inf. Th., vol. 41, pp. 216-232, Jan. 1995.

\section{Appendix A: Time Domain Parameters of Signal Cyclostationarity and Their Sample Estimates}

For a complex-valued continuous-time $n$ th-order cyclostationary process, $r(t)$, the $n$ th-order/ $q$-conjugate time-varying cumulant, $c_{r}(t ; \tau)_{n, q}$, is an (almost) periodic function of time, and can be expressed as an exponential Fourier series [26],

$c_{r}(t, \tau)_{n, q}=\sum_{\beta \in \kappa_{r, n, q}} c_{r}(\beta ; \tau)_{n, q} e^{j 2 \pi \beta t}$,

where the coefficient represents the $n$ th-order/ $q$-conjugate CC of $r(t)$ at CF $\beta$ and delay vector $\tau$, given by

$c_{r}(\beta ; \tau)_{n, q}=\lim _{I \rightarrow \infty} I^{-1} \int_{-I / 2}^{I / 2} c_{r}(t ; \tau)_{n, q} e^{-j 2 \pi \beta t} d t$.

The $n$ th-order/ $q$-conjugate time-varying cumulant is expressed in terms of the $n$ th- and lower-orders moments, through the moment to cumulant formula, as [26]

$$
\begin{aligned}
c_{r}(t ; \tau)_{n, q}= & \operatorname{Cum}\left[r^{(\cdot) 1}\left(t+\tau_{1}\right) \ldots r^{(\cdot)_{n-1}}\left(t+\tau_{n-1}\right) r^{(\cdot)_{n}}(t)\right] \\
& =\sum_{\left\{q_{1}, \ldots, \rho_{2}\right\}}(-1)^{Z-1}(Z-1) ! \prod_{z=1}^{Z} m_{r}\left(t ; \tau_{2}\right)_{n_{2}, q_{z}},
\end{aligned}
$$

where $(*)_{u}, u=1, \ldots, n$, represents a possible conjugation, so that the total number of conjugations is $q,\left\{\wp_{1}, \ldots, \wp_{Z}\right\}$ is a partition of $\wp=\{1,2, \ldots, n\}$, with $\wp_{z}$ 's, $z=1, \ldots, Z$, as non-empty disjoint subsets of $\wp$, so that their reunion is $\wp, Z$ is the number of the subsets in a partition $(1 \leq Z \leq n), \tau_{z}$ is a delay vector whose components are elements of $\left\{\tau_{1}, \ldots, \tau_{n-1}, \tau_{n}=0\right\}$, with indices specified by $\wp_{z}$, and $n_{z}$ is the number of elements in the subset $\wp_{z}$, from which $q_{z}$ correspond to conjugate terms. Note that $\sum_{z=1}^{z} n_{z}=n$ and $\sum_{z=1}^{Z} q_{z}=q$. Furthermore, $m_{r}\left(t ; \tau_{z}\right)_{n_{z}, q_{z}}$ is the $n_{z}$ th-order/ $q_{z}$ conjugate time-varying moment of $r(t)$, defined as 


$$
m_{r}\left(t ; \tau_{z}\right)_{n_{z}, q_{z}}=\mathrm{E}\left[r^{\left({ }^{(}\right), z}\left(t+\tau_{1, z}\right) r^{(*)_{2, z}}\left(t+\tau_{2, z}\right) \ldots r^{(*)_{n_{2}, z}}\left(t+\tau_{n_{z}, z}\right)\right]
$$

where $\tau_{v, z}, v=1, \ldots, n_{z}$, are the components of the delay vector $\tau_{z}$, and $(*)_{v, z}, \quad v=1, \ldots, n_{z}$, represents a possible conjugation, corresponding to the subset $\wp_{z}$ and the conjugate terms in (24), so that the total number of conjugations is $q_{z}$.

The $n$ th-order/ $q$-conjugate $\mathrm{CC}$ of $r(t)$ at $\mathrm{CF} \beta$ and delay vector $\tau$ can be expressed as [26]

$c_{r}(\beta ; \tau)_{n, q}=\sum_{\left\{\wp_{1}, \ldots, \wp_{Z}\right\}}(-1)^{Z-1}(Z-1) ! \sum_{\chi^{\dagger} 1=\beta} \prod_{z=1}^{Z} m_{r}\left(\chi_{z} ; \tau_{z}\right)_{n_{z}, q_{z}}$,

where $\chi=\left[\chi_{1} \ldots \chi_{Z}\right]^{\dagger}$ is a vector of CFs, 1 is a $Z$-dimensional one vector, and $m_{r}\left(\chi_{z} ; \tau_{z}\right)_{n_{z}, q_{z}}$ is the $n_{z}$ th-order/ $q_{z}$-conjugate cyclic moment of $r(t)$ at cycle frequency $\chi_{z}$ and delay vector $\tau_{z}$, defined as

$m_{r}\left(\chi_{z} ; \tau_{z}\right)_{n_{z}, q_{z}}=\lim _{I \rightarrow \infty} I^{-1} \int_{-I / 2}^{I / 2} m_{r}\left(t ; \tau_{z}\right)_{n_{z}, q_{z}} e^{-j 2 \pi \chi_{z} t} d t$.

Eq. (26) represents the so called cyclic moment to cumulant formula, which gives the $n$ th-order CCs as a function of the $n$ th- and lower-orders cyclic moments.

If the sampling rate $f_{s}$ fulfills the condition of no aliasing [26], the $n$ th-order/ $q$-conjugate $\mathrm{CCs}$ of the discrete-time signal $r(m)=\left.r(t)\right|_{t=m f_{s}^{-1}}$ are given by $\tilde{c}_{r}(\tilde{\beta} ; \tilde{\boldsymbol{\tau}})_{n, q}=c_{r}\left(\beta f_{s}^{-1} ; \tau f_{s}\right)_{n, q}$. A similar expression can be also written for cyclic moments [26]. Furthermore, (22)-(27) can be easily rewritten for discrete-time signals (see, for example, [27]). The estimate of the $n_{z}$ th-order/ $q_{z}$-conjugate cyclic moment based on $N_{s}$ samples is given by [27]

$$
\begin{aligned}
\hat{m}_{r}\left(\chi_{z} ; \tau_{z}\right)_{n_{2}, q_{z}} & =N_{s}^{-1} \sum_{m=1}^{N_{s}} r^{(*)_{1, z}}\left(m+\tilde{\tau}_{1, z}\right) r^{(*)_{2, z}}\left(m+\tilde{\tau}_{2, z}\right)_{\ldots} \\
& \times r^{(*)_{n_{z, z}}}\left(m+\tilde{\tau}_{n_{z}, z}\right) e^{-j 2 \pi \tilde{\chi}_{z} m},
\end{aligned}
$$

where $\tilde{\boldsymbol{\tau}}_{z}=\boldsymbol{\tau}_{z} f_{s}$, with its components $\tilde{\tau}_{v, z}=\tau_{v, z} f_{s}, \quad v=1, \ldots, n_{z}$, and $\tilde{\chi}_{z}=\chi_{z} f_{s}^{-1}$. Then, the estimate of the $n$ th-order/ $q$ conjugate CCs, based on $N_{s}$ observed samples, $\hat{c}_{r}(\beta ; \tau)_{n, q}$, is obtained by replacing the cyclic moment estimates in the cyclic moment to cumulant formula [27].

Eqs. (24)-(25) can be easily written for stationary processes, by dropping the $t$-dependency, and further simplified for random variables. Consider the variable $s_{l}$, with values taken from the alphabet corresponding to a signal constellation. The $n_{z}$ th-order/ $q_{z}$-conjugate moment of the constellation is defined as $m_{s, n_{z}, q_{z}}=\mathrm{E}\left[\left(s_{l}^{*}\right)^{q_{z}}\left(s_{l}\right)^{n_{z}-q_{z}}\right]=M^{-1} \sum_{m=1}^{M}\left(\tilde{s}_{m}^{*}\right)^{q_{z}}\left(\tilde{s}_{m}\right)^{n_{z}-q_{z}}$.

Then, by using the moment to cumulant formula, the $n$ thorder/ $q$-conjugate cumulant of the signal constellation, $c_{s, n, q}$, can be easily expressed in terms of moments as

$$
c_{s, n, q}=\operatorname{Cum}\left[\left(s_{l}^{*}\right)^{q}\left(s_{l}\right)^{n-q}\right]=\sum_{\left\{\wp_{1}, \ldots, \wp_{Z}\right\}}(-1)^{Z-1}(Z-1) ! \prod_{z=1}^{Z} m_{s, n_{z}, q_{z}} .
$$

\section{Appendix B: Cyclostationarity Test}

The cyclostationarity test developed in [23] is used for decisionmaking in signal detection and classification. The presence of a $\mathrm{CF}$ is formulated as a binary hypothesis-testing problem, i.e., under hypothesis $H_{0}$ the tested frequency $\beta^{\prime}$ is not a CF at delay $\tau$, and under hypothesis $H_{1}$ the tested frequency $\beta^{\prime}$ is a CF at delay $\tau$. The cyclostationarity test consists of the following three steps:

(1) - The $n$ th-order/ $q$-conjugate $\mathrm{CC}$ of the received signal is estimated (from $N_{s}$ samples) at tested frequency $\beta^{\prime}$ and delay $\tau$, and a vector

$\hat{\mathbf{c}}_{n, q}=\left[\operatorname{Re}\left\{\hat{c}_{r}\left(\beta^{\prime} ; \tau\right)_{n, q}\right\} \operatorname{Im}\left\{\hat{c}_{r}\left(\beta^{\prime} ; \tau\right)_{n, q}\right\}\right]$

is formed, with $\hat{c}_{r}\left(\beta^{\prime} ; \tau\right)_{n, q}$ as the CC estimates and $\operatorname{Re}\{\cdot\}$ and $\operatorname{Im}\{\cdot\}$ as the real and imaginary parts, respectively;

(2) - A statistic,

$\Psi_{n, q}=L \hat{\mathbf{c}}_{n, q} \hat{\Sigma}_{n, q}^{-1} \hat{\mathbf{c}}_{n, q}^{\dagger}$,

is computed for the tested frequency, $\beta^{\prime}$, and delay, $\tau$. Here -1 denotes the matrix inverse and $\hat{\Sigma}_{n, q}$ is an estimate of the covariance matrix of the real and imaginary parts of the $\mathrm{CC}$ estimates [23];

(3) - The test statistic $\Psi_{2,1}$, calculated for the tested frequency $\beta^{\prime}$ and delay $\tau$, is compared against a threshold $\Gamma$. If $\Psi_{2,1} \geq \Gamma, \beta^{\prime}$ is evaluated to be a CF at delay $\tau$; otherwise not. $\Gamma$ is set for a desired (asymptotic) probability of deciding that $\beta^{\prime}$ is a CF for the delay $\tau$, given that this is not, which is expressed as $\operatorname{Pr}\left\{\Psi_{2,1} \geq \Gamma \mid H_{0}\right\}$. Since $\Psi_{2,1}$ has an asymptotic chi-square distribution with two degrees of freedom under the hypothesis $H_{0}$ [23], $\Gamma$ can be obtained from tables of the chi-squared distribution for the desired (asymptotic) probability. 